\title{
Supporting and maintaining the frail patient on long-term renal replacement therapy
}

\author{
Authors: Louise Ryan ${ }^{\mathrm{A}}$ and Edwina Brown ${ }^{\mathrm{B}}$
}

The number of frail patients reaching end-stage kidney disease is increasing. They have a high level of comorbidity and symptom burden which need to be considered when making management plans. For those who choose renal replacement therapy, it is important to establish goals and ceilings of care and to provide holistic care focusing on optimising quality of life. Advance care planning is the process of documenting the patient's preferences for their treatment in the event they lose capacity to make decisions about their treatment.

DOI: $10.7861 /$ clinmed.2019-0416

\section{Introduction}

With our ageing population, the numbers of older patients requiring renal replacement therapy (RRT) are increasing. This population brings a high level of complexity related to their high number of comorbidities, including frailty. Frailty has been defined as a physiologic state of increased vulnerability to stressors that results from decreased physiologic reserves or dysregulation of multiple physiologic systems. ${ }^{1}$ Recognised prevalence among incident dialysis patients is up to $73 \%$. $^{2}$ Frailty is not, however, the preserve of older dialysis patients; a high prevalence is also found among younger patients with $63 \%$ of patients under the age of 40 years who require RRT meeting the criteria for frailty in one study. ${ }^{2}$ RRTs used in frail patients include in-centre haemodialysis (HD), peritoneal dialysis (PD) and kidney transplantation. Given the lack of evidence that dialysis improves survival of frail older patients with end-stage kidney disease (ESKD) many will opt for maximal conservative management. ${ }^{3}$ This includes active treatment of anaemia, fluid status and symptoms of uraemia without resorting to dialysis. Establishing the goal of RRT with the patient and relatives, be it rehabilitative with an aim towards transplantation (though this is unlikely in the majority of frail older patients), life sustaining or symptom relieving, will help direct appropriate treatment, interventions and supportive care.

Authors: ${ }^{A}$ post-CCT nephrology fellow, Imperial College Renal and Transplant Centre, London, UK; ${ }^{\text {B }}$ rofessor of renal medicine, Imperial College London, London, UK and consultant nephrologist, Imperial College Renal and Transplant Centre, London, UK

\section{Assessing for frailty}

The first step to addressing frailty in our ESKD population is identifying it. The European Renal Best Practice guidelines for managing older patients with chronic kidney disease recommend that this cohort, including those requiring RRT, be screened regularly to identify those who would benefit from a formal geriatric assessment. ${ }^{4}$ They acknowledge there is insufficient evidence to recommend one individual score to assess this cohort but recommend simple scores which include self-reporting scales and functional screens, including gait speed and sit-to-stand tests. Once identified, individualised exercise programmes, which include both strength and endurance elements, can have a positive effect on frail patients' functional status. ${ }^{4}$ Exercise such as cycling can even be incorporated into haemodialysis sessions. As with all frail individuals admitted to hospital with acute illness, early mobilisation of patients with ESKD is important to retain mobility.

\section{Supportive care}

Patients with advanced kidney disease, particularly frail patients who require RRT have a high burden of physical and psychosocial

\section{Key points}

Frailty is a syndrome that not only effects older patients receiving renal replacement therapy but can also be present in up to $63 \%$ of patients under 40 years old who receive renal replacement therapy (RRT).

Establishing the goal of the patient's RRT can help to appropriately direct treatments and interventions.

Cognitive impairment is common in patients receiving RRT and is often under recognised.

Frail patients with end-stage kidney disease can have a high burden of physical and psychosocial symptoms which may persist despite adequate RRT.

Altered pharmacokinetics and pharmacodynamics make frail older patients particularly susceptible to drug-drug interactions; polypharmacy should therefore be avoided.

KEYWORDS: Frailty, haemodialysis, peritoneal dialysis, cognitive impairment, supportive care 
symptoms with a shortened life expectancy. The median survival of patients over 75 years old commencing RRT is approximately 2 years in the UK. ${ }^{5}$ Symptoms can include pain, nausea, pruritus, depression, anxiety, shortness of breath, restless leg syndrome and sleep disturbances and can be comparable to those experienced by patients with malignancy. ${ }^{6}$ These symptoms are usually multifactorial and often persist despite adequate dialysis. Supportive care, which aims to improve quality of life through early identification, assessment and treatment of symptoms, should be integral to the management of all frail patients with ESKD and not restricted to those who opt for conservative care or those withdrawing from dialysis. ${ }^{7}$ Likewise, palliative care input should be sought for frail individuals with difficult to manage symptoms throughout their RRT journey and not just as part of end-of-life care.

\section{Planning for the future}

Central to the supportive care process is the prioritisation of the individual's values and preferences. The process of documenting these values to outline a plan for future health and personal care in the situation of no longer having capacity to make decisions is termed advance care planning (ACP). ACP can improve end-of-life care, improve patient satisfaction and emotional distress in surviving family members when optimally utilised. ${ }^{8}$ Despite the frequent healthcare interactions this cohort has and hence many opportunities for ACP discussions, ACP is underutilised. ${ }^{9}$ Awareness of and engagement with ACP are increasing among nephrologists and, given the longitudinal relationship between nephrologists and their patients, ACP discussions with their primary nephrologist can be invaluable. Adverse clinical events or deterioration in chronic conditions should trigger a conversation, revisiting the patient's preferences to ensure that their current treatment remains aligned to their goals of care and may include discussion of withdrawal of dialysis. A decision to withdraw dialysis should be shared between the patient, family and treating team with a plan for end-of-life care agreed. Withdrawal of dialysis precedes death in approximately $36 \%$ of patients receiving dialysis. ${ }^{10}$

\section{Cognitive impairment}

Cognitive impairment is common in patients with ESKD and is often undiagnosed. In a study of adult haemodialysis patients, only $28.9 \%$ of patients had no impairment of cognitive function in any domain. ${ }^{11}$ The most common domains impaired were in complex attention (48.8\%), learning and memory ( $42.2 \%)$ and in executive function (41.7\%). This can result in difficulties in fully engaging with their treatment plans and in decision making. For this reason, ACP and encouraging patients to identify a spokesperson in the event they lose capacity in the future should be encouraged. The rate of decline in cognitive function appears to be greater in patients receiving HD compared to PD. ${ }^{12}$ This may be secondary to intermittent cerebral hypoperfusion experienced during HD sessions and thus every practical effort needs to be taken to prevent this. ${ }^{13}$ It is important to screen for cognitive impairment in this population and assess for potential reversible causes. Depression, which is also common in patients with ESKD with a prevalence up to $30 \%$, can mimic dementia and hence needs to be ruled out and treated as appropriate. ${ }^{14}$

\section{Blood pressure, fluid balance and medications}

The optimal target blood pressure for patients receiving HD has not been well established. Treatment and targets need to be individualised particularly in the frail and older population. $\mathrm{HD}$ patients with low pre-dialysis systolic blood pressure and orthostatic hypotension have an increased risk of falls, which are associated with a higher risk of fractures and mortality than the general population. ${ }^{15}$ All hypertensive frail patients requiring RRT should have a standing blood pressure checked prior to treating with antihypertensive medication. Overtreating hypertension in patients receiving dialysis with antihypertensive medications can make it more difficult to remove excess fluid on dialysis and lead to patients developing resistant hypertension secondary to sodium and water retention. Large interdialytic fluid gains may require extra $\mathrm{HD}$ sessions to remove the fluid to reduce the risk of intradialytic hypotension caused by high ultrafiltration rates which can result in cerebral hypoperfusion and cognitive decline. ${ }^{13}$ All patients admitted to hospital who receive RRT should be discussed with their primary renal team and dialysis unit to ensure a continuity of their therapy. Older, multimorbid and frail patients are often exposed to polypharmacy which increases inappropriate drug-drug interactions and adverse events. Patients with advanced kidney disease are particularly vulnerable due to altered pharmacokinetics and pharmacodynamics. ${ }^{16}$ Great care needs to be taken in prescribing drugs in frail patients requiring RRT to ensure that drugs are properly adjusted for RRT modality and to avoid drug-drug interactions particularly with kidney transplantation immunosuppressive drugs.

\section{Nutrition}

Nutritional advice and dietary supplements prescribed must be tailored to the individual's treatment goals. The standard electrolyte and fluid restricted diets that are prescribed to patients on RRT may not be in keeping with the goals of a frail patient whose treatment targets are quality of life rather than prolongation of life. The dietary strategy utilised should support the patient to make informed diet-related decisions, reduce food-related anxiety and incorporate cultural values and social aspects of eating with counselling from a registered dietitian with experience in this area. ${ }^{17}$ The syndrome of frailty and chronic kidney disease is associated with poor dietary intake and sarcopenia. Exercise programmes designed to maintain and promote independence in frail patients should be supported by a higher energy, proteincontrolled diet under the supervision of a dietitian. ${ }^{17}$ The end of life is often associated with a reduced appetite and desire to drink. Patients and their families should be reassured to reduce anxiety regarding nutritional intake and following dietary restrictions and the patient encouraged to focus on foods most enjoyed. ${ }^{17}$

\section{Modality of RRT}

When deciding between conservative kidney management or between the different modalities of RRT, the patient, their family and treatment team must keep values and treatment goals at the forefront. For those who decide to commence dialysis, they must be counselled on the advantages and disadvantages of both $\mathrm{HD}$ and PD. There is no clear survival benefit for older patients with either modality compared to the other. ${ }^{18}$ The Frail Elderly Patient Outcomes on Dialysis (FEPOD) study showed better treatment satisfaction for patients on assisted PD compared to HD; the principal association with quality of life, though, was frailty. ${ }^{19}$ Assisted PD, which enables patients who cannot perform their own PD to receive PD by providing healthcare personnel or by training family to assist them, is available in many European 
countries, Canada and Australia. ${ }^{20}$ Other factors that patients should consider include location of dialysis (hospital or home), travel, impact on social activities and family life. The patient's nephrologist should individualise the prescription of dialysis (frequency and duration) to the goals of care while minimalising treatment burden. ${ }^{21}$

\section{Conflicts of interest}

Prof Edwina Brown has received speaker fees and has been on the advisory board for Baxter Healthcare.

\section{References}

1 Fried LP, Ferrucci L, Darer ] et al. Untangling the concepts of disability, frailty, and comorbidity: implications for improved targeting and care. J Gerontol Med Sci 2004;59:255-63.

2 Bao Y, Dalrymple L, Chertow GM et al. Frailty, dialysis initiation, mortality in end-stage renal disease. Arch Intern Med 2012;172:1071-7.

3 Verberne WR, Geers AB, Jellema WT et al. Comparative survival among older adults with advanced kidney disease managed conservatively versus with dialysis. Clin J Am Soc Nephrol 2016;11:633-40

4 Farrington K, Covic A, Aucella F et al. Clinical Practice Guideline on management of older patients with chronic kidney disease stage 3b or higher (eGFR $<45 \mathrm{~mL} / \mathrm{min} / 1.73 \mathrm{~m}^{2}$ ). Nephrol Dial Transplant 2016;31(suppl 2):ii1-66.

5 Steenkamp R, Pyart R, Fraser S. Chapter 5: Survival and cause of death in UK ADULT patients on renal replacement therapy in 2016. Nephron 2018;139(Suppl 1):117-50.

6 Saini T, Murtagh FE, Dupont P] et al. Comparative pilot study of symptoms and quality of life in cancer patients and patients with end stage renal disease. Palliat Med 2006;20:631-6.

7 Davison SN, Levin A, Moss AH et al. Executive summary of the KDIGO controversies conference on supportive care in chronic kidney disease: developing a roadmap to improving quality care. Kidney Int 2015;88:447-59.

8 Detering KM, Hancock AD, Reade MC et al. The impact of advance care planning on end of life care in elderly patients: randomised controlled trial. BMJ 2010:230:1345.

9 Feely MA, Hildebrandt D, Edakkanambeth Varayil J, Mueller PS. Prevalence and contents of advance directives of patients with ESRD receiving dialysis. Clin J Am Soc Nephrol 2016;11:2204-9.
10 Findlay MD, Donaldson K, Doyle A et al. Factors influencing withdrawal from dialysis: a national registry study. Nephrol Dial Transplant 2016;31:2041-8.

11 van Zwieten A, Wong G, Ruospo M et al. Prevalence and patterns of cognitive impairment in adult hemodialysis patients: the COGNITIVE-HD study. Nephrol Dial Transplant 2018;33:1197-206.

12 Wolfgram DF, Szabo A, Murray AM et al. Risk of dementia in peritoneal dialysis patients compared with hemodialysis patients. Perit Dial Int 2015;35:189-98.

13 Polinder-Bos HA, Vállez García D, Kuipers ] et al. Hemodialysis induces an acute decline in cerebral blood flow in elderly patients. JASN 2018:29:1317-25.

14 Fischer MJ, Porter AC, Lash JP. Treatment of depression and poor mental health among patients receiving maintenance dialysis: are there options other than a pill or a couch? Am J Kidney Dis 2013:61:694-7.

15 Morley JE. Online Curricula: Geriatric Nephrology: Chapter 33: Falls in elderly patients with kidney disease. American Society of Nephrology, 2009. www.asn-online.org/education/distancelearning/curricula/geriatrics

16 Morin L, Vetrano DL, Rizzuto D et al. Choosing wisely? Measuring the burden of medications in older adults near the end of life: Nationwide, longitudinal cohort study. Am J Med 2017;130:927-36.e9.

17 Stevenson J, Meade A, Randall AM et al. Nutrition in renal supportive care: patient-driven and flexible. Nephrology 2017;22:739-47.

18 Harris SA, Lamping DL, Brown EA et al. Clinical outcomes and quality of life in elderly patients on peritoneal dialysis versus hemodialysis. Perit Dial Int 2002;22:463-70.

19 Iyasere $\mathrm{O}$, Brown EA, Johansson $\mathrm{L}$ et al. Quality of life and physical function in older patients on dialysis: a comparison of assisted peritoneal dialysis with hemodialysis. CJASN 2016;11:423-30.

20 Brown EA, Wilkie M. Assisted peritoneal dialysis as an alternative to in-center hemodialysis. CJASN 2016;11:1522-4.

21 Vandecasteele SJ, Kurella Tamura M. A patient-centered vision of care for ESRD: dialysis as a bridging treatment or as a final destination? JASN 2014;25:1647-51.

Address for correspondence: Dr Louise Ryan, Nephrology Department, Hammersmith Hospital, Du Cane Road, London W12 0HS, UK.

Email: louise.ryan6@nhs.net 